Meta

Journal des traducteurs

Translators' Journal

\title{
Lire et traduire les "silences " du texte : manifestations isotopiques à travers un poème de Rimbaud et ses traductions en anglais
}

\section{Iulia Mihalache}

Volume 47, numéro 4, décembre 2002

URI : https://id.erudit.org/iderudit/008032ar

DOI : https://doi.org/10.7202/008032ar

Aller au sommaire du numéro

Éditeur(s)

Les Presses de l'Université de Montréal

ISSN

0026-0452 (imprimé)

1492-1421 (numérique)

Découvrir la revue

Citer cet article

Mihalache, I. (2002). Lire et traduire les « silences » du texte : manifestations isotopiques à travers un poème de Rimbaud et ses traductions en anglais. Meta, 47(4), 479-497. https://doi.org/10.7202/008032ar
Résumé de l'article

La traduction est l'ombre de l'original, son complément, son actualisation, sa plénitude. Pour recréer ce « souvenir circulaire » qui fait qu’un texte porte en lui et retourne incessamment vers une multitude d'autres textes, nous devons nous situer non seulement au niveau du texte en soi, mais aussi dans l'espace de l'intertextualité et de l'interdiscursivité. L'analyse des manifestations isotopiques à travers le poème " Les Chercheuses de poux " d'Arthur Rimbaud et ses diverses traductions en anglais nous permet de reconstituer un ensemble de représentations individuelles et collectives, manifestes ou implicites : l'impensé de l'auteur et de son époque, tout comme le « silence » de la culture qui traduit. 


\title{
Lire et traduire les «silences » du texte: manifestations isotopiques à travers un poème de Rimbaud et ses traductions en anglais
}

\author{
IULIA MIHALACHE \\ Université d'Ottawa, Ottawa, Canada
}

\section{RÉSUMÉ}

La traduction est l'ombre de l'original, son complément, son actualisation, sa plénitude. Pour recréer ce «souvenir circulaire » qui fait qu'un texte porte en lui et retourne incessamment vers une multitude d'autres textes, nous devons nous situer non seulement au niveau du texte en soi, mais aussi dans l'espace de l'intertextualité et de l'interdiscursivité. L'analyse des manifestations isotopiques à travers le poème «Les Chercheuses de poux» d'Arthur Rimbaud et ses diverses traductions en anglais nous permet de reconstituer un ensemble de représentations individuelles et collectives, manifestes ou implicites: l'impensé de l'auteur et de son époque, tout comme le «silence» de la culture qui traduit.

\begin{abstract}
Translation is the shadow or the counterpart of the original, its coming into being, its fulfillment. To recreate this "circular souvenir," which makes a text nourish other texts or endlessly come back to them, we must go beyond the text, towards intertextuality and interdiscursivity. The analysis of the different types of isotopy present throughout Rimbaud's poem «Les Chercheuses de poux» and its English translations allows us to reconstitute a series of individual and collective representations, either manifest or implicit: the "incorporeal discourse" of the author and his epoch, as well as the "silence" of the translating culture.
\end{abstract}

\section{MOTS-CLÉS/KEYWORDS}

isotopie manifeste, isotopie métaphorique, isotopie de l'expression, isotopie du contenu, isotopie textuelle

[...] all manifest discourse is based on an 'already-said'; and [that] this 'already-said' is not merely a phrase that has already been spoken, or a text that has already been written, but a 'never-said,' an incorporeal discourse, a voice as silent as a breath, a writing that is merely the hollow of its own mark. It is supposed therefore that everything that is formulated in discourse was already articulated in that semi-silence that precedes it, which continues to run obstinately beneath it, but which it covers and silences ${ }^{1}$.

\section{INTRODUCTION}

Un texte est transparent et secret à la fois. Installé dans une réalité, dans une certaine 'objectivité', de par son appartenance à un auteur et à un espace-temps régissant ses règles de production et de réception, le texte organise et transpose cette réalité dans sa texture même. En même temps un texte détient une composante d'indétermination [...] qui permet la participation du lecteur à la production de l'intention du texte (Iser 
1985 : 55). Réplique à des textes passés ou bien précurseur d'idées et thèmes à caractère public (culturel), un texte partage son savoir avec d'autres textes et se définit ainsi par une cohérence intertextuelle. L'espace textuel cependant n'est pas non plus singulier, mais participe à la production discursive 'totale' d'une société. Tout en restant inédit, c'est-à-dire une perspective individuelle d'imaginer le monde, un texte établit un dialogue imaginaire avec d'autres discours qui circulent à travers les époques et les sociétés, pour devenir le produit d'une culture ou, de nos jours, d'une interculture. À l'intérieur de celle-ci, les perspectives individuelles multiples construisent, par l'itération de problématiques communes, une sorte de point de repère, d'espace de reconnaissance de ce qui fait la spécificité de la culture / de l'interculture respective et de ce qui définit chacun de ses membres. Le texte se définit aussi par une cohérence interdiscursive.

La notion d'isotopie est à la fois signe de la transparence et de l'inédit du texte. Définie comme étant un réseau sémantique constitué d'au moins deux éléments, disséminés dans le récit et qui partagent des sèmes communs, l'isotopie peut être manifeste, renvoyant à un ensemble de traits sémantiques permanents, non modifiables à travers plusieurs textes (sèmes nucléaires) ou bien métaphorique, indiquant une certaine vision de l'énonciateur immergé dans un espace socioculturel. L'isotopie se retrouve ainsi dans le texte, au niveau de l'expression (graphique, phonologique, syntaxique) ainsi qu'au niveau du contenu (bien qu'une isotopie d'expression puisse produire une isotopie du contenu), à travers les textes et dans les échanges interdiscursifs.

À partir du poème «Les Chercheuses de poux » d'Arthur Rimbaud, nous allons analyser les différentes isotopies textuelles, manifestes ou métaphoriques, et la façon dont elles sont recréées par une série de traducteurs anglais: Ezra Pound², Enid Rhodes Peschel ${ }^{3}$, Jeremy Reed ${ }^{4}$, Wallace Fowlie ${ }^{5}$, John Theobald ${ }^{6}$ et Robert Lowell ${ }^{7}$. En même temps, nous allons voir comment le texte original révèle certains thèmes récurrents à travers l'œuvre du poète symboliste Rimbaud (l'isotopie intertextuelle) et comment ces thèmes sont en harmonie avec l'esprit du temps (l'isotopie interdiscursive). L'isotopie interdiscursive traverse les genres et les époques et nous fait retrouver la peinture de Léon Zack, intitulée aussi "Les Chercheuses de poux", peinture qui semble répondre au désir de Rimbaud d'écrire dans une langue «des sons et des parfums».

\section{LE TEXTE COMME GESTE DU POÈTE FACE AU «COSMOS ${ }^{8}$}

Le rapprochement entre les événements de la vie de Rimbaud et le poème Les Chercheuses de poux, publié en 1870, conduit vers une lecture "factuelle» ou purement informative du texte. Le 19 juillet 1870 la France déclare la guerre à la Prusse. À Charleville, la ville natale de Rimbaud qui n'a alors que 16 ans, tout est à feu et à sang et le jeune poète, ne voyant plus d'espoir de survie à l'art, s'enfuit à Paris. Il s'y fait arrêter pour avoir voyagé sans billet et, suspecté d'être du camp des Prussiens, il est transporté à la prison de Mazas. Relâché, grâce à son professeur Georges Izambard, il va à Douai pour rester deux semaines chez son professeur et trois «charmantes» vieilles filles de sa parenté (les demoiselles Gindre). Les «trois vieilles filles », réalité transposée et modifiée dans le poème («deux grandes sœurs»), représente un premier indice historique (Mailloux 1995: 124) qui pourrait être utilisé par le lecteur dans l'interprétation allégorique, symbolique du poème. À cet indice s'ajoutent 
d'autres faits réels de la vie du poète: orphelin de père, sa mère l'étouffe par son exigence tout au long de son enfance; au collège, il est tout d'abord catholique fervent pour se révolter après contre Dieu; il aime le grec et le latin et n'hésite pas à écrire ses premiers poèmes en latin. En reprenant la terminologie de Rastier (1972: 85), il s'agirait, lors de cette lecture préliminaire, de la découverte de deux « isotopies horizontales», appelée aussi isotopies sémémiques. La première est manifeste parce qu'elle évoque une réalité (la guerre franco-allemande); elle peut être désignée par le mot «guerre». Cette isotopie est très intéressante parce qu'elle contient aussi des éléments qui sont allégoriques pour qui ne connaît pas les événements réels qui entourent l'écriture du poème (voir notre explication plus loin). La deuxième isotopie est manifeste parce que les sémèmes qui la composent ne nécessitent pas d'explication supplémentaire du point de vue sémantique; elle peut être désignée par le mot «épouillage». C'est surtout la deuxième isotopie qui fait ressortir la nécessité d'une lecture plus approfondie, métaphorique du poème, parce qu'elle ne peut pas «expliquer» la présence des associations visuelles, sonores, de rime et de rythme, établies entre les éléments du poème.

\section{(1) Première isotopie horizontale: LA GUERRE}

/chercheuses de poux/: des femmes qui enlèvent la saleté du 'prisonnier' (ici: les demoiselles Gindre) OU des personnes qui sont à la recherche, à la poursuite de quelqu'un qui se cache comme les poux (les Prussiens contre les Français).

/enfant/: le jeune Rimbaud sorti de la prison, affaibli

/plein de rouges tourmentes/: le poète affligé par des souffrances physiques ou morales /rouges/: le sang que la Guerre fait couler. En rapport avec la "guerre», «rouge » évoque aussi l'idée d'un soldat blessé. En faisant le lien avec un autre poème de Rimbaud, l'enfant, c'est le dormeur du val: «Un soldat jeune, bouche ouverte, tête nue, / Et la nuque baignant dans le frais cresson bleu, / Dort; il est étendu dans l'herbe, sous la nue, / Pâle dans son lit vert où la lumière pleut».

/tourmentes/: les troubles politiques / sociaux violents et profonds, qui affectent le pays

/rêves/: le désir de Paix

/son lit/: le possessif «son» dessine l'image de la «maison» en contraste avec celle de la «prison» qui exclue toute idée de "propriété». Pour aller plus loin, la "prison» dépouille le prisonnier de tout bien.

/essaim/: la "fuite» de Charleville (essaim renvoie au désir de s'établir ailleurs) et la sortie de la prison

/essaim/: les troupes de soldats

/blanc/, /argentins/: la Paix

/bleu/, /blanc/, /rouge/: le drapeau de la France

/sœurs/: celles qui soignent les malades, les blessés, les prisonniers

/croisée/ : la Guerre, par son association avec croisade. Selon Le Petit Robert, «croisade» est un mot formé par réfection du mot «croisée».

/croisée/: la fenêtre de la cellule du prisonnier; c'est la tentation de la liberté (l'air bleu) qui rend cette fenêtre "grande ouverte»

/l'air bleu/: la liberté

/fouillis/: le désordre qui règne dans le pays

/tombe/: les soldats qui tombent ou qui meurent

/terribles/, /craintifs/: l'horreur de la guerre

/sifflement/: le sifflement d'appel à la guerre

/battant/: «battre» = «canonner», frapper de projectiles 
/crépiter/: ce sont les mitrailleuses qui crépitent

/royaux/: renvoie au roi de la Prusse, Guillaume $1^{\text {er }}$. Note: dans la traduction de Jeremy Reed, la référence à la royauté est plus explicite; Reed traduit ongles royaux par sovereign nails.

/mort/, /mourir/: conséquence de la guerre

/vin/, /harmonica/, /délirer/: fêter la fin de la guerre (qui n'est qu'un rêve pour l'ins$\operatorname{tant}$ )

/pleurer/: déception, amertume provoquées par la guerre

(2) Deuxième isotopie horizontale: L'ÉPOUILLAGE

/chercheuses de poux/: des femmes (sœurs infirmières, sœurs de charité) qui épouillent un /enfant/ quelconque

/plein de rouges tourmentes/: souffrance (allant jusqu'à la fièvre) provoquée par les poux

/essaim blanc/: la propreté

/indistincts/: effet de la fièvre

/lit/: l'enfant est couché parce que la sensation de malaise est trop forte

/sœurs/: infirmières; suggère aussi l'idée de "propreté» (les infirmières sont habillées en blanc)

/ongles argentins/: la propreté

/croisée/: la fenêtre: elle fait entrer la lumière et facilite la «recherche de poux»

/fouillis/: même si l'image s'applique à la nature, le mot établit la correspondance avec l'idée de "cheveux sales et emmêlés»

/lourds cheveux/: les cheveux sont pleins de poux

/promènent leurs doigts/ : épouillent

/leurs haleines craintives/: la peur et le dégoût suscités par l'épouillage

/crépiter/ + /mort des petits poux/: les poux qui meurent

/vin de la paresse/: euphorie due au fait d'avoir retrouvé la propreté

Nous constatons qu'il y a différents sémèmes qui sont situés sur les deux isotopies à la fois. Cependant, quand certains sémèmes d'une isotopie «peuvent être lus sur l'autre isotopie, ils le sont parce qu'on suspend à la lecture les sèmes caractéristiques de leur isotopie et que l'on supplée ceux de l'autre» (Rastier 1972: 88). Ainsi, «crépiter» renvoie dans la première isotopie aux mitrailleuses et dans la deuxième au bruit fait par l'écrasement des poux.

La première isotopie horizontale exige, pour qu'elle soit actualisée lors de la lecture, une connaissance a priori du cadre social de production du poème (la guerre franco-allemande). La connaissance des réalités de guerre peut rendre cette isotopie manifeste dans la traduction, comme elle peut la laisser implicite, métaphorique (voir notre analyse des isotopies métaphoriques de l'original et des traductions). Nous constatons aussi que, si cette isotopie peut fonctionner comme point de départ dans une approche symbolique (allégorique) du poème, elle n'est pas toujours prise en compte. Parfois elle peut être perdue de vue non pas parce qu'elle serait de surcroît, mais parce que le lecteur ou le traducteur, en fonction de ses compétences culturelles, croit juste d'activer certains aspects plus que d'autres, c'est-à-dire de rendre certaines isotopies dominantes par rapport à d'autres. Le traducteur Robert Lowell ne voit plus les sœurs comme étant 'charmantes', mais pareilles à des meurtrières. Mises en contraste avec des fées qui viendraient secourir l'enfant, les «sœurs» dans cette nouvelle image donnent au poème une connotation supplémentaire: l'isotopie sémémique de la guerre est remplacée dans la traduction par l'isotopie dominante 
dans un conte, qui est basée sur la dichotomie bon-mauvais. La structure profonde de cette isotopie ou son schéma actantiel (Greimas 1966), c'est le récit nucléaire à partir duquel sont engendrés les personnages et les actions visibles à la surface du récit:

le SUJET de la quête, celui qui cherche, veut obtenir ou désire quelque chose:/child/

l'OBJET de la quête ou ce qui est recherché: /his life/

l'ADJUVANT ou celui qui aide le sujet dans sa quête: /fairies/

l'OPPOSANT ou celui qui entrave le sujet dans sa quête: /royal sisters/

le DESTINATEUR (une force ou un être qui motive la quête du sujet): /his ceaseless wish to cry/

le DESTINATAIRE ou le bénéficiaire immédiat ou ultime de la quête réalisée par le sujet: absent

Ce schéma génère deux paradigmes distincts, celui du bien ou de la lumière (Child, fairies, a glass of violets, blue, singing breath, scent of rose, wine of idleness, harmonica, rise), et celui du mal ou du ténébreux (feverish, frowning, red, knife, wicked razor fingers, tried to breathe, dark, struck, crush, bloated, blood, disdainful, flushed his eyes, sigh, insanely, wearied lungs, dying, cry). C'est la dominance du deuxième paradigme (il compte plus d'éléments que le premier) qui donne au poème une teinte mystérieuse dans le sens négatif

Ces observations nous permettent de dire que la reconstruction du contexte socioculturel est un facteur primordial dans l'analyse d'un texte et de ses traductions. Il nous semble ainsi plus raisonnable de replacer tout d'abord le poème «Les Chercheuses de poux» dans l'ensemble des discours de l'époque (isotopie interdiscursive), pour voir après comment il fait corps commun avec l'œuvre de Rimbaud (isotopie intertextuelle), tout en préservant son originalité (isotopie textuelle). Ces trois perspectives nous permettrons de faire une lecture tabulaire du texte, durant laquelle nous reviendrons sur nos pas pour découvrir ou bien pour anticiper de nouvelles significations du texte. Notre lecture sera ainsi une suite de lectures possibles.

\section{LES ÉCHOS DE L'ÉPOQUE. TEXTE ORIGINAL ET TRADUCTIONS}

L'«ombre» (Barthes 1973: 53) qui accompagne toujours un texte amène le lecteur dans un espace où chaque dire en fait résonner un autre; c'est, comme le dit toujours Barthes, une espèce de "souvenir circulaire» qui fait que l'on vit toujours dans une intertextualité: "Ce "moi" qui s'approche du texte est déjà lui-même une pluralité d'autres textes, de codes infinis, ou plus exactement: perdus (dont l'origine se perd) » (Barthes 1970: 16).

Le discours de Rimbaud est, dans un certain sens, un reflet de son époque puisque sa pensée est comprise dès le départ dans les structures poétiques du Discours romantique. Les thèmes de l'amour, de l'évasion, de l'exil, de la révolte, de la dualité de l'être humain et de l'osmose avec la Nature, qui sont présents dans la création de Rimbaud, engendrent une relation dialogique entre l'œuvre individuelle et l'ensemble à l'intérieur duquel elle se situe. C'est ce qui nous permet de voir, par exemple, certaines ressemblances entre Rimbaud et Nietzsche (qui publie en 1871 Naissance de la tragédie à partir de l'esprit de la musique) : le thème de l'équivalence entre la création et l'ivresse, l'élément dionysiaque comme noyau d'une musique qui atteint les profondeurs du monde, la musique et le plastique comme fondements de la poésie et 
puissance de l'art et de l'être humain. Cependant Rimbaud est aussi celui qui se veut moderne, qui refuse de s'inféoder à la pensée de son époque. Il construit à l'intérieur de l'«idéologie dominante» un langage-système (Meschonnic 1970: 354), c'est-à-dire un corps de symboles et de formes littéraires qui assurent la lisibilité, l'unité et l'inédit de son ouvre.

Pour relier cette réflexion à la démarche de Pound qui entreprend de traduire le poème de Rimbaud, nous rappelons que dans l'introduction aux Essais Littéraires d'Ezra Pound, T.S. Eliot fait voir le rôle joué par Pound dans le contexte de son époque. Il s'agit d'une aptitude à «dire ce qu'il faut dire» à un moment de l'histoire, c'est-à-dire à répondre aux attentes explicites ou implicites de sa culture ; cela explique pourquoi Pound avait choisi de commenter les œuvres des auteurs et des littératures jusque là négligés, mal compris ou mésestimés.

Ce sont les mêmes Essais Littéraires (1954) qui nous fournissent les principes de base de tout acte d'écriture ou de traduction, tel qu'il est conçu par Ezra Pound. En ce qui concerne la traduction des poèmes de Rimbaud, elle devrait suivre la mesure, la clarté et la précision des Anciens; ce type de traduction jouerait sur la relation archaïque-savant parce que: "In Rimbaud the image stands clean, unencumbered by non-functioning words; to get anything like this directness of presentation one must go back to Catullus [...]»(1954:33).

Les trois types de poésie énoncés par Pound (1954: 25) viennent appuyer sa démarche traductive. La Mélopée - «wherein the words are charged, over and above their meaning, with some musical property» — se découvre dans le rythme du poème (le calme se transforme en agitation) et dans la multitude d'allitérations: réitération du son «s» (lice, «sibilant, the saliva's hiss», kiss, "squiffer's sigh»), du son «f» ( «forehead is full of», "wafty fingers»), du son « $\mathrm{r} »$ ( «Imploring swarms of dreams», «large and charming sisters», «run fine, alluring, terrible/Fingers through », " "Crack!" to break») ou réitération de deux sons à la fois ( «so soft fingers death assails»). Pound même nous donne la clé de ses allitérations en utilisant le mot sibilant qui signifie à la fois sibilant et fricatif (dont l'articulation comporte un resserrement du canal vocal, comme dans $f, z$, s, et th [en anglais]). La Phanopée "which is a casting of images upon the visual imagination" — a trait à la traduction des associations inédites entre les mots, qui poussent l'esprit dans les zones de l'imagination les plus éloignées: "l'air bleu baigne un fouillis de fleurs", "cils noirs battant sous les silences parfumés", "grises indolences", etc. La Logopée — «the dance of the intellect among the words" — vise la reconstitution personnelle, originale, de l'ensemble des symboles mis en marche par le poète, symboles qui représentent la quintessence de sa pensée et de la pensée de son époque.

Le choix de Pound de donner à sa traduction un air plus intellectuel (surtout par l'emploi de mots et de syntagmes archaïques, comme anon, Lo) ne signifie pas un désir d'abstraction. Cela ne l'empêche pas d'utiliser un terme argotique comme squiffer ou de faire appel à une structure prosodique moderne (rejets par rapport au texte de départ, parmi lesquels: "And run fine, alluring, terrible/Fingers through his thick dew-matted hair»). Pound croit en fait à la puissance de tout mot d'exprimer un complexe intellectuel et émotionnel. L'impression faite sur le lecteur doit être fulgurante ( It is better to present one Image in a lifetime than to produce voluminous works») et pour cette raison la traduction ne devrait pas rester au niveau de l'enchaînement syntaxique des mots, mais se soucier au contraire du rythme interne d'un 
texte. La traduction de Pound contraste ainsi avec la traduction littérale (mot à mot) de Wallace Fowlie.

\section{LE CARACTÈRE UNITAIRE DE L'GUVRE. RÉCURRENCE DES THÈMES}

On découvre à travers l'espace poétique de Rimbaud une pluralité de figures, d'images et de symboles qui constituent, à tour de rôle, un des Masques du 'moi' rimbaldien. À partir de l'idée que l'isotopie a une définition syntagmatique, mais non syntaxique (Rastier 1972: 82) — ce qui veut dire qu'elle est un ensemble non ordonné du point de vue syntaxique, mais ordonné du point de vue rhétorique et stylistique - nous pouvons dire que chaque poème rimbaldien peut être vu comme un prolongement ou comme une anticipation d'un autre: les éléments textuels se ressemblent et se reflètent les uns dans les autres.

\section{L'enfant}

Dans l'un de ses premiers poèmes, «Les Étrennes des orphelins », Rimbaud introduit déjà le thème de l'enfant malheureux, dépourvu d'amour maternel et devenu mûr avant l'âge: les pensées le dévorent et ses rêves ne sont plus quiets. L'enfant est à la quête de la maternité, qui aboutira à la douleur de se sentir seul pour toujours: «Et, tout pensifs, tandis que de leurs grands yeux bleus / Silencieusement tombe une larme amère, / Ils murmurent: 'Quand donc reviendra notre mère?' ». Les premiers thèmes sont là: l'enfant pensif, orphelin ou oublié, le lit maternel (champ de l'intimité), le rêve, les pleurs. L'enfant se désignera dans «Les Poètes de sept ans » comme le poètegénie, le poète qui apporte la modernité. D’ailleurs Rimbaud décrit souvent l'enfant en termes de capacité intellectuelle (fréquence du front comme élément descriptif).

L'indigence affective équivaut à un exil de sa propre enfance ou bien à une projection différente de ce qu'est l'enfance: le côté érotique de l'enfance marque une évolution anormale ou trop rapide de l'amour maternel et c'est le thème du désir agressif, fou (dans «Enfance»). La douleur de l'exil entraîne le sacrifice de soi-même (thème du sang). On retrouve à cet instant l'image de l'innocence accompagnée de la marque christique; l'enfant c'est le Fils de Dieu et le front rouge signifie le front blessé par les épines: "L'Enfant qui ramassa les balles, le Pubère / Où circule le sang de l'exil et d'un Père» («L'Enfant qui ramassa...»). L'allusion au Christ est encore visible dans d'autres poèmes, où s'insère en même temps l'image de la Vierge Marie, signe de maternité et de féminité. D'après Bayo (1987: 87), "l'homme au front rouge », c'est un esclave qui vient d'être libéré, donc un enfant qui, à la manière de Rimbaud, a retrouvé sa liberté par un exil de sa propre enfance.

L'enfant se présente toujours en train de rêver, de penser ou bien d'errer à travers les sentiers; il n'agit pas: "l'action n'est pas la vie, mais une façon de gâcher quelque force, un énervement», dit Rimbaud dans «Délires». L'acte de travailler équivaut à une impureté parce qu'il prive le poète de la vérité; il n'y a qu'un seul travail qui compte et c'est celui de la création poétique, du travail de l'esprit («L'Impossible»).

Le travail de création est pourtant un sacrifice et il ne laisse au poète que les sensations: «Je ne sais plus parler» («Matin»). Le travail de l'esprit, celui qui motive son désir d'«être absolument moderne»(«Adieu»), a un seul but: «Je veux être 
poète, et je travaille à me rendre Voyant» (Correspondance, 13 mai 1871). Le poète cherche en fait ce qui est abstrait, ce qui est censé contenir l'essentiel; cette quête ne se réalise pas par les mots, mais par les sensations.

Le renoncement à la parole entraîne une exploitation au maximum de tous les sens: "Il s'agit d'arriver à l'inconnu par le dérèglement de tous les sens». Mallarmé, dans ses Euvres complètes, affirme lui-aussi: "Nommer un objet, c'est supprimer les trois-quarts de la jouissance du poème qui est faite de deviner peu à peu: le suggérer, voilà le rêve» (Mallarmé 1974: 869). Les tourmentes rouges qui traversent le front de l'enfant dans «Les Chercheuses de poux» peuvent ainsi être vues comme un résultat de ce désir de capter et de transmettre l'ineffable: "J'écrivais des silences, des nuits, je notais l'inexprimable. Je fixais des vertiges. [...] Je finis par trouver sacré le désordre de mon esprit. J'étais oisif, en proie à une lourde fièvre», dit Rimbaud dans «Délires ».

L'isotopie, à son tour, ne vise pas à nommer, mais à offrir des indices de lectures, compris comme "sèmes", "attributs récurrents", qui aident à la configuration d'UNE lecture possible, qui n'est quand même pas la seule.

\section{La femme}

Les personnages féminins des «Chercheuses de poux» ont l'air d'êtres évanescents, de contours: "grandes », « charmantes », «frêles doigts », « ongles argentins », « doigts fins, terribles et charmeurs», "cils noirs», " ongles royaux». Leur portrait, basé sur une série d'antinomies (grandes / frêles, charmantes / terribles, argentins / noirs) suggère la dualité de la femme (aspect présent également dans Illuminations), ce qui explique aussi pourquoi il s'agit de «deux» sœurs au lieu de trois.

Chez Rimbaud, la femme est signe d'une perte de la foi, elle est femme de société, elle est aussi la Mère, la Vierge. Mais il y a toujours un aspect religieux qui s'insère dans sa description. L'infidèle avait été originairement promise au salut (comme «La Vierge folle»), une autre est en train de goûter les plaisirs mondains, alors qu'au début elle était pure ${ }^{9}$. Une autre fois, la femme est Sœur (sœur religieuse ou sœur de quelqu'un), elle s'associe parfois à la fleur, donc à la pureté naturelle, mais la fleur semble contenir un poison: «Et les atroces fleurs qu'on appellerait cœurs et sours» («Métropolitain»; nous soulignons).

L'amour pour l'être féminin revêt lui-aussi un double aspect: d'un côté, c'est le bonheur d'un amour harmonieux qui assure l'équilibre, la chasteté du Moi poétique, de l'autre, c'est l'amour brutal, animal. L'agressivité de l'amour que l'on retrouve dans la troisième strophe des «Chercheuses de poux» («[...] salives / reprises sur la lèvre ou désir de baisers») est encore illustrée dans «Tête de faune», où l'éros innocent s'affronte à l'éros charnel.

\section{L'ivresse}

Aux images du sang et de l'amour ensanglanté évoquées ci-dessus s'ajoutent les symboles du vin, de la soif et de l'ivresse. Nous découvrons dans «Les Chercheuses de poux» trois actants principaux: l'enfant, les sœurs et la Paresse (soulignons l'emploi de la majuscule dans le texte original) ou «le vin de la Paresse». La présence du vin évoque celle de l'eau (la rivière, comme dans «Le Bateau ivre»), des pleurs (larmes) et de la soif. 
La Paresse, l'absence de l'action, est, comme nous l'avons vu, préparatrice pour le travail poétique. Elle prédispose au rêve qui n'est pas doux, mais affaiblissant, parce qu'il consomme toutes les énergies du poète. Rêver, c'est synonyme de boire: «Puis, quand j'ai ravalé mes rêves avec soin, / Je me retourne, ayant bu trente ou quarante chopes», dit Rimbaud dans «Oraison du soir». L'acte de boire devient une purification si l'absorption du poison en est le déclencheur: «Il [le poète] cherche lui-même, il épuise en lui tous les poisons, pour n'en garder que les quintessences » (Correspondance, 15 mai 1871). L'inoculation du poison comme méthode nécessaire dans le "dérèglement de tous les sens » a été fournie à Rimbaud par les Paradis artificiels de Baudelaire, qui voyait dans le haschisch l'occasion d'une «évaporation lente, successive, éternelle» de l'esprit («Le Poème du haschisch»). L'ivresse provoquée par le «désordre de l'esprit» s'accompagne toujours d'une obligation de tristesse, d'une souffrance. Les pleurs constituent une étape poétique décisive dans la création: «Je pleurais énormément, à tout cela... Enfin... j’ai laissé finir toutes les larmes de mon corps avec cette nuit... Vrai, cette fois j'ai pleuré plus que tous les enfants du monde» («Déserts de l'amour»).

\section{LES ISOTOPIES TEXTUELLES : ORIGINAL ET TRADUCTIONS}

Après avoir considéré quelques-unes des images symboliques récurrentes à travers l'œuvre de Rimbaud, nous analyserons les isotopies métaphoriques de l'original et de ses traductions sans prétendre d'épuiser les significations du poème et sans vouloir impliquer que le poème serait redondant par rapport aux autres poèmes rimbaldiens. Nous remarquerons que certaines isotopies sont conflictuelles et que cette tension est constitutive de l'originalité du poème. Les conflits d'ordre sémantique génèrent des contradictions dans l'esprit du lecteur et le poussent à chercher des significations possibles, potentielles. Les isotopies constituent des ensembles sémémiques à travers tout le poème ou à travers des parties du poème. Le changement d'isotopie signifie la possibilité d'une nouvelle interprétation.

L'isotopie de l'enfance heureuse entre en contraste l'isotopie de la maturité maladive de l'enfant. L'enfant écoute, entend ou bien sent: trois verbes qui ne comportent apparemment rien de nuisible ou de dangereux, mais qui, ensemble avec d'autres éléments, construisent l'image d'un enfant dévoré et tué par ses pensées. 


\begin{tabular}{|c|c|c|}
\hline $\begin{array}{l}\text { Enfance heureuse } \\
\text { (sèmes positifs) }\end{array}$ & Observations & $\begin{array}{l}\text { Maturité maladive } \\
\text { (sèmes négatifs) }\end{array}$ \\
\hline /enfant/ & $\begin{array}{l}\text { Enfant peut être situé dans l'isotopie de l'enfance } \\
\text { heureuse parce que la représentation que l'on se fait } \\
\text { habituellement de l'enfance comporte des attributs } \\
\text { dominants comme joie, manque de soucis, sérénité. } \\
\text { Le verbe throb («The child's head throbs»), situé dans la } \\
\text { traduction de Reed dans le cadre de la deuxième } \\
\text { isotopie, rend plus violente l'idée de maladie. L'état de } \\
\text { l'enfant est anormal, convulsif. En plus, le traducteur } \\
\text { focalise son attention et celle du lecteur sur la tête de } \\
\text { l'enfant (head) et non pas sur le front (forehead). } \\
\text { Dans la traduction de Theobald, tout comme dans celle } \\
\text { de Peschel, l'enfant est tantôt child, tantôt boy. «Boy» } \\
\text { masculinise l'enfant et perd de vue l'idée de passivité que } \\
\text { Rimbaud associe habituellement à l'image de l'enfant. }\end{array}$ & /front/ \\
\hline /blanc/ & $\begin{array}{l}\text { Pound accentue dans sa traduction l'idée de capacité } \\
\text { cognitive de l'enfant, en remplaçant tourmentes par } \\
\text { contents. } \\
\text { Reed traduit tourmentes par flush, sémème connoté à la } \\
\text { fois positivement et négativement. Quand placé dans la } \\
\text { première isotopie, flush exprime «la rougeur au visage } \\
\text { vu comme signe de bonne santé». Cette interprétation } \\
\text { est possible vu le sémème qui suit, à savoir spark } \\
\text { (signifiant «lueur d'esprit»). Quand flush fonctionne } \\
\text { sur la deuxième isotopie, il exprime «une réponse } \\
\text { somatique aux blessures ou aux irritations, caractérisée } \\
\text { par la douleur, l'enflure, la rougeur et la fièvre». Cette } \\
\text { deuxième interprétation n'est pas non plus exclue vu le } \\
\text { deuxième sémème qui suit, à savoir pain. En ce qui } \\
\text { concerne le mot spark, il établit la correspondance avec } \\
\text { electrical plus loin dans le poème, pourvu que spark soit } \\
\text { lu comme «émission d'étincelles». Si placé dans } \\
\text { l'isotopie de la guerre, spark pourrait signifier le début } \\
\text { d'une révolution (spark off veut dire «déclencher une } \\
\text { révolution»). } \\
\text { Dans la traduction de Lowell, l'accent est mis sur la } \\
\text { fièvre et la colère de l'enfant: The child, feverish, } \\
\text { frowning, only saw red. }\end{array}$ & /rouges tourmentes/ \\
\hline & $\begin{array}{l}\text { Rêves et dreams peuvent être connotés à la fois } \\
\text { positivement et négativement. Du point de vue négatif, } \\
\text { rêve signifie imagination délirante, alors que dream } \\
\text { renvoie à un fantasme produit par l'inoculation d'une } \\
\text { drogue. } \\
\text { Theobald construit pour «rêves indistincts» une image } \\
\text { tout à fait contraire: the white swarm of dreams that sleep } \\
\text { unveils. On voit pourtant dans les œuvres des poètes } \\
\text { symbolistes que l'idée de "voile» (veil), de "voiler» et } \\
\text { «dévoiler» (unveil) est très fréquente; le lecteur est }\end{array}$ & \\
\hline
\end{tabular}




\begin{tabular}{|c|c|c|}
\hline /rêves/ & $\begin{array}{l}\text { quelqu'un qui déchiffre, qui enlève le voile (unveil), qui } \\
\text { découvre l'inconnu (tout comme l'enfant le fait } \\
\text { progressivement). Poe dit dans «The Veil of the Soul»: } \\
\text { "Were I called on to define, very briefly, the term 'Art', I } \\
\text { should call it 'the reproduction of what the Senses perceive } \\
\text { in Nature through the veil of the soul'» (Poe cité par } \\
\text { Peschel } 1981: 1 \text { ), alors que Yeats, dans «The Trembling of } \\
\text { the Veil», nous fournit d'autres indices sur les } \\
\text { symbolistes, par l'allusion à Baudelaire: "I have found in } \\
\text { an old diary a saying from Stéphane Mallarmé, that his } \\
\text { epoch was troubled by the trembling of the veil of the } \\
\text { Temple» (Yeats cité par Peschel 1981: 47). } \\
\text { Reed aussi transpose dans sa traduction une même } \\
\text { image centrée sur l'idée de « voile» (he dreams of faces } \\
\text { behind streaming veils), la différence étant qu'il se place } \\
\text { dans l'isotopie négative et imagine l'enfant en train } \\
\text { d'avoir des hallucinations. Streaming, l'adjectif qui } \\
\text { accompagne veils, renvoie à la fois à l'idée de sang qui } \\
\text { coule (à cause d'une maladie ou à cause d'une blessure } \\
\text { causée par la guerre) et à l'idée de mouvement en masse } \\
\text { (comme le mouvement des troupes ou comme le } \\
\text { déplacement des abeilles; streaming établit donc la } \\
\text { correspondance avec swarms qui, à son tour, peut } \\
\text { transmettre: la fuite de Rimbaud de la prison, les troupes } \\
\text { de soldats ainsi que la multitude de poux). } \\
\text { Les rêves indistincts, dans la traduction de Peschel, } \\
\text { deviennent dreams with hazy details. L'utilisation de } \\
\text { details permet de se placer doublement dans l'isotopie de } \\
\text { la guerre analysée plus haut: (1) employé au pluriel, } \\
\text { details signifie une information confidentielle; (2) } \\
\text { employé au singulier, detail peut signifier, entre autres, } \\
\text { « détachement» ( petit groupe de soldats détachés du } \\
\text { gros de la troupe pour un service spécial»). Dans les } \\
\text { deux cas, detail et details peuvent donc contenir le sème } \\
\text { «militaire». }\end{array}$ & $\begin{array}{l}\text { /rêves/ } \\
\text { /indistincts/ }\end{array}$ \\
\hline$/$ lit/ & $\begin{array}{l}\text { Le lit maternel (connotation positive) contraste avec le lit } \\
\text { d'un malade (connotation négative). Pound se situe dans } \\
\text { la première isotopie; il agrandit l'espace d'intimité par } \\
\text { l'emploi de bedroom. }\end{array}$ & /lit/ \\
\hline /sœurs/ & $\begin{array}{l}\text { Sceurs, ainsi que rêves, lit, charmantes, charmeurs, frêles, } \\
\text { peut recouvrir deux sémèmes différents opposés, dans le } \\
\text { cas de sœurs, par la catégorie «lien de parenté / sans lien } \\
\text { de parenté ». Cette opposition en crée une autre } \\
\text { supplémentaire: affection } v s \text {. froideur. Søurs engendre } \\
\text { donc des lectures plurivoques; cependant, c'est le } \\
\text { contexte ou la dominance d'un certain contexte qui } \\
\text { imposera une certaine interprétation. Sœurs peut donc } \\
\text { connoter positivement le sentiment de tendresse } \\
\text { maternelle que les deux femmes inspirent à l'enfant, tout } \\
\text { comme sœurs peut connoter négativement le sentiment } \\
\text { de peur inspiré par l'arrivée des infirmières. }\end{array}$ & /sœurs/ \\
\hline
\end{tabular}




\begin{tabular}{|c|c|c|}
\hline /charmantes/ & $\begin{array}{l}\text { Sémème qui renvoie, en français et en anglais, à la fois à } \\
\text { la captation de l'intérêt de l'enfant et à sa séduction } \\
\text { magique, sorcière. }\end{array}$ & /charmantes/ \\
\hline /frêles/ & $\begin{array}{l}\text { Si frêles exprime la finesse de l'enfant, il bloque l'isotopie } \\
\text { à l'intérieur de laquelle frêles signifierait « dépourvu } \\
\text { d'énergie, faible, malade». Ce conflit sémantique n'est } \\
\text { pas présent dans la traduction de Pound (wafty connote } \\
\text { la gentillesse, la douceur), alors que Reed et Lowell } \\
\text { traduisent frêles par long. }\end{array}$ & /frêles/ \\
\hline /argentins/ & $\begin{array}{l}\text { Argentin peut tout d'abord connoter la candeur de } \\
\text { l'enfant. Métaphoriquement, argentin ou silvery peut } \\
\text { aussi renvoyer à un son clair (idée d'euphonie): le } \\
\text { sémème établit ainsi une correspondance avec le verbe } \\
\text { chanter et entre en conflit avec sifflement qui exprime un } \\
\text { son à la fois aigu et intense. } \\
\text { Ongles est connoté négativement non seulement par les } \\
\text { sèmes qui le composent, mais aussi par son apparition à } \\
\text { côté du mot argentin. Ongles s'inscrit dans le paradigme } \\
\text { «lame, griffe, serre», ce qui explique pourquoi Lowell } \\
\text { traduit ongles par couteau et pourquoi il est le seul à } \\
\text { mentionner les dents là où Rimbaud (et les autres } \\
\text { traducteurs aussi) parle de lèvre (lips). Ongles peut } \\
\text { contenir le sème «aigu», ce qui le met en rapport avec } \\
\text { sifflement. }\end{array}$ & /ongles/ \\
\hline $\begin{array}{l}\text { /l'air bleu baigne } \\
\text { un fouillis de } \\
\text { fleurs/ } \\
\text { /tombe la rosée/ }\end{array}$ & $\begin{array}{l}\text { Les deux syntagmes expriment une atmosphère paisible } \\
\text { et joyeuse qui entoure l'enfant. Seul fouillis introduit une } \\
\text { teinte négative (v. l'isotopie de la laideur plus loin). } \\
\text { Blue en anglais peut exprimer la tristesse. } \\
\begin{array}{l}\text { Dans la traduction de Lowell, les fleurs deviennent } \\
\text { violets, ce qui fait le lien avec l'idée de royauté (le } \\
\text { pourpre, c'est la couleur royale), que nous avons énoncée } \\
\text { dans l'analyse de l'isotopie de la guerre. }\end{array}\end{array}$ & $\begin{array}{l}\text { /fouillis/ } \\
\text { /blue/ [anglais] }\end{array}$ \\
\hline $\begin{array}{l}\text { /promènent leurs } \\
\text { doigts fins }[\ldots] \\
\text { charmeurs/ }\end{array}$ & $\begin{array}{l}\text { La connotation négative de charmeur est explicite dans la } \\
\text { traduction de Peschel qui traduit charmeur par bewitch- } \\
\text { ing, et encore plus dans la traduction de Lowell qui } \\
\text { exclut toute idée de charme magique: wicked razor } \\
\text { fingers. } \\
\text { Plusieurs traducteurs traduisent le verbe promener, qui } \\
\text { connote un sentiment de plaisir, par des verbes plutôt } \\
\text { négatifs qui s'inscrivent soit dans l'isotopie de la guerre } \\
\text { soit dans celle de la maladie: probe (chez Reed) a trait à } \\
\text { une fouille ou à un examen médical minutieux; march } \\
\text { (chez Lowell) peut indiquer la marche des troupes de } \\
\text { soldats; run (chez Pound) évoque la fuite. }\end{array}$ & $\begin{array}{l}\text { /terribles/ } \\
\text { /charmeurs/ }\end{array}$ \\
\hline & & $\begin{array}{l}\text { /il écoute/, /il } \\
\text { entend/, /il sent }\end{array}$ \\
\hline
\end{tabular}




\begin{tabular}{|c|c|c|}
\hline /chanter/ & $\begin{array}{l}\text { Le verbe chanter exprime le cadre harmonieux dans } \\
\text { lequel l'enfant est placé. Cette idée est maintenue dans la } \\
\text { traduction de Pound, par exemple, qui traduit le chant } \\
\text { par rustling («caractérisé par des sons doux»), alors que } \\
\text { Reed se place du côté de l'isotopie négative: vibrates fait } \\
\text { ainsi le lien avec throbs du début du poème dans la } \\
\text { même traduction. }\end{array}$ & \\
\hline & $\begin{array}{l}\text { L'angoisse exprimée par craintives n'est pas présente } \\
\text { dans la traduction de Pound ou dans celle de Theobald } \\
\text { (timid). Fowlie maintient ce sème et en ajoute un autre } \\
\text { supplémentaire qui exprime la maturité de l'enfant: } \\
\text { apprehensive signifie à la fois «inquiet, angoissé», mais } \\
\text { aussi «qui a un bon jugement, qui perçoit bien les } \\
\text { choses». }\end{array}$ & /haleines craintives/ \\
\hline \multirow[t]{4}{*}{$\begin{array}{l}\text { /qui fleurent de } \\
\text { longs miels } \\
\text { végétaux et rosés/ }\end{array}$} & $\begin{array}{l}\text { Végétaux évoque la couleur verte, donc la force de la } \\
\text { nature, l'énergie. Miel, comme honey, exprime l'affection. } \\
\text { Miel établit la correspondance avec essaim et renvoie en } \\
\text { même temps à la couleur d'ambre (jaune doré). Le sème } \\
\text { "ambre», présent dans le sémème miel, établit la } \\
\text { correspondance avec silences parfumés, et avec électrique, } \\
\text { l'ambre jaune étant une résine qui a la propriété de } \\
\text { s'électriser par frottement. } \\
\text { Si anon, dans la traduction de Pound, est compris } \\
\text { comme «anone, pomme cannelle» (substantif) et non } \\
\text { pas comme «at another time» (adverbe), il entre alors en } \\
\text { relation avec miel. }\end{array}$ & \\
\hline & $\begin{array}{l}\text { Sifflement, tout comme hiss en anglais (traduction de } \\
\text { Pound), exprime le malaise, le mécontentement. }\end{array}$ & /sifflement/ \\
\hline & Peut connoter une maladie. & /salives/ \\
\hline & $\begin{array}{l}\text { Baiser est connoté négativement surtout parce qu'il } \\
\text { apparaît à côté de lèvre et d'enfant. Lowell va plus loin } \\
\text { dans sa traduction pour présenter le tout comme un fait } \\
\text { accompli: the lips he kissed. }\end{array}$ & /désirs de baisers/ \\
\hline $\begin{array}{l}\text { /les silences } \\
\text { parfumés/ }\end{array}$ & $\begin{array}{l}\text { Alors que Pound et Theobald se maintiennent dans la } \\
\text { première isotopie (quiétude, hush), Lowell opère un } \\
\text { déplacement vers la deuxième isotopie: in the dark. Reed } \\
\text { aussi connote négativement le sentiment d'une émotion } \\
\text { violente: Beat like frenetic moths. }\end{array}$ & $\begin{array}{l}\text { /cils noirs [qui } \\
\text { battent]/ }\end{array}$ \\
\hline /leurs doigts doux/ & $\begin{array}{l}\text { Electriques peut être encadré dans la deuxième isotopie à } \\
\text { cause du sème "violent» («de l'intensité d'une décharge } \\
\text { électrique»). Dans la traduction de Lowell, ce sème est } \\
\text { repris par le passé struck: electric finger struck to crush. }\end{array}$ & /électriques/ \\
\hline /indolences/ & $\begin{array}{l}\text { L'adjectif grises connote la tristesse, alors qu'indolences } \\
\text { exprime un état d'apathie, d'insensibilité. Indolences } \\
\text { (chez Pound) connote la passivité, l'aversion pour le } \\
\text { travail. Leaden, qui est la traduction pour grises chez } \\
\text { Theobald, évoque un enfant dépourvu de joie, d'éclat. }\end{array}$ & /grises indolences/ \\
\hline
\end{tabular}




\begin{tabular}{|c|c|c|}
\hline & $\begin{array}{l}\text { Indolences, tout comme Paresse, peut être placé dans le } \\
\text { cadre de la première isotopie seulement si l'on se } \\
\text { rappelle que Rimbaud refuse tout travail qui n'est pas un } \\
\text { travail de création, un travail poétique. }\end{array}$ & \\
\hline /paresse/ & $\begin{array}{l}\text { La Paresse peut signifier une lenteur de réaction } \\
\text { anormale (deuxième isotopie). Idleness, dans la traduc- } \\
\text { tion de Lowell, exprime le mépris du travail. Sloth (chez } \\
\text { Fowlie) transpose les sèmes de "paresse» mais comporte } \\
\text { aussi des sèmes supplémentaires: «Apathy and inactivity } \\
\text { in the practice of virtue (personified as one of the deadly } \\
\text { sins)». }\end{array}$ & /paresse/ \\
\hline /harmonica/ & $\begin{array}{l}\text { L'harmonie d'une enfance heureuse, évoquée par } \\
\text { harmonica, contraste avec la douleur et l'état de maladie } \\
\text { exprimés par soupir (sigh). Dans la traduction de Reed, le } \\
\text { syntagme deluding wail (traduction de soupir) introduit } \\
\text { des sèmes supplémentaires: deluding signifie trompeur; } \\
\text { wail signifie cri plaintif. }\end{array}$ & /soupir/ \\
\hline $\begin{array}{l}\text { /la lenteur des } \\
\text { caresses/ } \\
\text { /délirer/ }\end{array}$ & $\begin{array}{l}\text { Les caresses expriment l'affection, alors que délirer } \\
\text { connote à la fois la joie et le trouble. Delirium (traduc- } \\
\text { tion de Pound) renvoie à un état d'agitation mentale } \\
\text { violente. Lowell connote la folie et l'étouffement: } \\
\text { insanely, wearied lungs. } \\
\text { Les caresses construisent une autre image dans la } \\
\text { traduction de Reed qui exprime l'insensibilité de } \\
\text { l'enfant: dulled by wine. }\end{array}$ & /délirer/ \\
\hline \multirow[t]{2}{*}{ /sourdre/ } & $\begin{array}{l}\text { Pound se place dans la deuxième isotopie seulement: } \\
\text { «Wane and fade» exprime une disparition graduelle. }\end{array}$ & /mourir/ \\
\hline & $\begin{array}{l}\text { Désir de pleurer établit la symétrie avec désir de baisers } \\
\text { (isotopie syntaxique). } \\
\text { Dans la traduction de Reed, le désir de pleurer est un } \\
\text { renouveau spirituel: a future lifting sail. Reed opère donc } \\
\text { un changement d'isotopie par rapport à l'original. }\end{array}$ & $\begin{array}{l}\text { /Désir de pleurer/ } \\
\text { — symétrie avec } \\
\text { désir de baisers }\end{array}$ \\
\hline
\end{tabular}

L'isotopie de la prière et du rêve de chasteté (paradigme biblique) est construite, dans l'original, par «implore», «blanc», «rêves», "sœurs» (signifiant "religieuses»), «croisée» (qui peut évoquer une église), «silences parfumés» (le syntagme évoque le recueillement et le parfum de l'encens), "désir de pleurer», auxquels on peut ajouter «rouges» comme symbole du sang et du sacrifice. La rime « rosée croisée», absente des traductions, peut renvoyer à la Rose-Croix, mouvement ésotérique et mystique qui croit à la religion (la croix) révélée par l'initiation à la science, à l'alchimie (conquête de la rose). Rimbaud même se voulait un poète voyant. Quelques observations s'imposent par rapport aux traductions: (1) Lowell maintient la référence de croisée à une église par l'emploi du sémème $\operatorname{arch}$; on peut dire que cette traduction évoque l'image de l'homme au front rouge (le Christ) par l'introduction du nom blood (sang) et du verbe mark qui peut signifier balafrer; le syntagme disdainful touch présent dans cette même traduction exprime une aversion pour le 
péché; (2) Fowlie met en relief l'idée de péché mortel pour l'utilisation de Sloth ( «apathy and inactivity in the practice of virtue [personified as one of the deadly sins»]); (3) Reed et Theobald imaginent les sœurs comme étant des religieuses, surtout par l'emploi du mot, et respectivement de la racine veil; (4) La référence aux religieuses dans la traduction de Reed est contradictoire à cause de l'utilisation de l'adjectif possessif: his two big sisters; (5) Des sémèmes qui apparaissent seulement dans la traduction de Reed rendent l'isotopie de la prière dominante par rapport aux autres isotopies de la prière, présentes dans l'original et dans le reste des traductions: unruly implique la désobéissance et contraste avec le syntagme he lies submissively, expire (rendre le dernier soupir) contraste avec awakenings (compris comme le fait d'être ressuscité).

L'isotopie de l'espace et de l'exil dans l'imagination est configurée par essaim (white swarm of dreams pour Theobald, fairies pour Lowell, mais connoté négativement chez Reed: he dreams of faces behind streaming veils), rêves (comme moyen d'évasion), croisée grande ouverte (wide-open window pour Pound et Fowlie, a casement opened wide pour Peschel, a dormer window pour Reed, a window view pour Theobald, mais diminution de l'espace chez Lowell: the window's arch, halfopen), l'air bleu, promènent (stroll pour Theobald, mais connotant la tension chez les autres traducteurs: run pour Pound et Peschel, probing pour Reed, move pour Fowlie, march pour Lowell). Croisée grande ouverte peut aussi évoquer l'image du poète novateur Rimbaud qui se trouve au carrefour des traditions. Une nouvelle image apparaît dans la traduction de Reed, sans qu'elle n'ait de correspondant dans le poème original: a future lifting sail.

L'isotopie de l'éveil n'est pas amplement illustrée, mais elle nous ramène à l'importance de l'éveil de l'esprit dans la recherche de la pureté: "Ces bons soirs de septembre où je sentais des gouttes / De rosée à mon front, comme un vin de vigueur», lit-on dans le poème rimbaldien Ma bohème. À la rosée qui évoque l'aube, s'ajoutent sourdre (absent de la traduction de Pound) ainsi que d'autres images qui nous font croire que l'enfant est en train de s'éveiller (Implore l'essaim blanc des rêves indistincts, grises indolences). Awakenings, présent dans la traduction de Reed, peut figurer également dans cette isotopie. L'image de l'aube est mise aussi en évidence par Pound: and "Crack!" to break his inebriated indolences.

L'isotopie de l'éros innocent, qui se retrouve dans le réseau sémantique lit (connote le lit maternel), sœurs charmantes, enfant, s'oppose à l'isotopie de l'éros charnel, illustrée par haleines craintives, $\underline{\text { sifflement, }} \underline{\text { salives, }} \underline{\text { lèvre, }}$ désirs de baisers, lenteur des

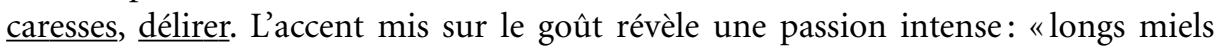
végétaux et rosés ». Nous remarquons que la traduction de Reed devient beaucoup plus érotique dans la quatrième strophe: "electric fingers set his blood on fire, / he lies submissively...» et que l'adjectif deluding qui figure dans la dernière strophe fait référence à un amour trahi. Les ongles, auxquels s'ajoutent les dents dans la traduction de Lowell, évoquent un amour féroce.

L'isotopie du désordre est présente dans le poème pour soutenir l'idée de "dérèglement de tous les sens»; le désordre se fait voir non seulement dans l'esprit (rouges tourmentes, rêves indistincts, délirer), mais aussi dans la nature: un fouillis de fleurs. Theobald traduit "fouillis de fleurs" par clumps of flowers; clump qui signifie non seulement cluster, mais aussi a mass of bacteria, élargit ainsi l'isotopie de la laideur dont l'analyse suit. 
L'isotopie de la laideur (poux, lourds cheveux signifiant «cheveux pleins de poux», petits poux, essaim signifiant «multitude de poux») renvoie à l'esthétique de la laideur, créée par Baudelaire, pour indiquer une prédilection pour la perversion (dans le sens d'une déviation de ce qui est conventionnellement accepté, du latin per et vertere $=$ se tourner), un désir de modernité (vouloir choquer) et une façon de percevoir la beauté dans ce qui est traditionnellement considéré comme répulsif. Baudelaire dit dans "Au Lecteur»: "Aux objets répugnants nous trouvons des appas». Lourd, dans le sens de "plein de poux», perd cette signification dans la traduction de Pound (thick hair) et dans celle de Theobald (unruly hair, signifiant « cheveux emmêlés»).

L'isotopie de la musique et de la danse, qui renvoie à la recherche des sensations et à l'amour de l'esprit grec, est tout d'abord perçue dans le rythme du poème et dans le rythme des gestes des deux sœurs (elles viennent près du lit, elles assoient l'enfant, elles promènent leurs doigts dans les cheveux de l'enfant). À cela s'ajoutent l'image de la rosée qui tombe, le «chant» des haleines, le sifflement, le «soupir», les cils noirs qui «battent» (comme le cœur, ou comme le tambour) et les caresses continuelles. L'harmonica ( «instrument de musique composé de petits tuyaux à anche métallique juxtaposés que l'on fait vibrer par le souffle») actualise plusieurs des thèmes de Rimbaud. Il évoque l'intention du poète d'écrire dans une langue «des sons et des parfums» (harmonika en grec signifie «théorie de la musique»). L'ancien sens du mot "harmonica"10 renvoie à l'image de l'eau vue comme endroit ou comme méthode de purification dans le travail créatif. L'harmonica suggère aussi l'image de la bouche (qui va de pair avec «lèvre», figurant dans l'isotopie de l'éros charnel) et il se rattache à ses autres correspondants dans la poésie, qui évoquent le souffle. L'isotopie phonique créée par « un sifflement, salives reprises sur la lèvre ou désirs de baisers» (alternance $\mathrm{s} / \mathrm{z}$ ) est transposée dans la traduction de Pound (sibilant, the saliva's hiss, / drawn from alip, or a desire to kiss) qui ajoute d'autres isotopies phoniques (mentionnées en début de cet article). Pound remplace l'image de l'harmonica par squiffer, qui signifie «ivrogne», ce qui élargit l'isotopie de l'ivresse (connotation négative) et bloque l'isotopie de la danse, de la musique, de la joie et la sérénité: quelqu'un peut être ivre de joie, mais il est impossible qu'il soit ivrogne de joie. Plus précisément, l'isotopie de l'ivresse chez Pound entre en conflit avec l'isotopie de la musique (du rythme), cette dernière comprenant également le sémème wafty (dans wafty fingers), qui évoque l'idée de flottement. Theobald utilise pour "harmonika» un mot qui n'a pas la même teneur symbolique — mouth-organ —, mais qui préserve la référence à la «bouche».

Les allitérations dans la traduction de Pound construisent des isotopies d'expression (isotopies phoniques) qui, à leur tour, produisent des isotopies de contenu. Lice, qui apparaît dans le titre de la traduction d'Ezra Pound, introduit la consonne s, répétée plusieurs fois à travers le poème et rendue explicite par le sémème sibilant. Mais plus que cela, le $s$ itératif évoque l'épuisement provoqué par la quête, l'étouffement, la perte de souffle et finalement la mort. Cette intensité des sentiments qu'ils soient rêves, fantasmes ou hantises - , nous rappelle la force des peintures de Francisco Goya pour qui el sueno de la razon produce monstruos ( «le sommeil de la raison produit des monstres»).

L'isotopie de l'ivresse euphorique suit celle de la musique. La chromatique du poème exploite le rouge dès le début ("plein de rouge tourmentes», red, red flush). 
Elle évolue vers « rosés» (pinkish, pink, rose, rosy, roses comme substantif) pour suggérer à la fin le rouge intense du vin. «Rosés» n’est pas séparé du «vin » (la formule elliptique «du rosé » signifie en fait «du vin rouge clair»), tout comme «paresse » est un résultat de l'ivresse, raison pour laquelle «Paresse» (avec ses diverses traductions: Laziness, forgetfulness, Sloth) entre dans cette isotopie. L'isotopie de l'ivresse euphorique inclut également le verbe "délirer» (renvoie à Dionysos) et l'adjectif «grises» (dans le sens de "légèrement ivre») dont la signification devient explicite dans la traduction de Pound (inebriated) et dans celle de Fowlie (half-drunken).

L'isotopie de la mort apporte au poème une teinte anti-auratique. La mort marque la tension douloureuse qui est inséparable de la création. Elle est annoncée graduellement: «lourds», «terribles», «charmeurs» (connoté négativement), «craintives», «noirs» (qui contraste avec «blanc», "argentins»), «la mort des petits poux», "soupir», "mourir» (qui contraste avec "sourdre» dans le sens de "naître $)^{11}$. Threat [menace] of delirium élargit ce réseau sémantique, dans la traduction de Theobald. Chez Theobald, tout comme chez Peschel, l'approche de la mort a aussi quelque chose de magique: «Stroll their slim fingers, magical with fear » et, respectivement, their slender fingers, terrible and bewitching. Reed construit le poème sur toute une série de verbes et d'expressions figées qui suggèrent l'approche d'un danger imminent, mortel (throbs, sparks, they begin their search, probing, vibrates, shiver, miss, catch, beat, set his blood on fire, lies submissively, crackles, expire), danger qui à la fin s'avère être une expérience qui provoque l'émotion, peut-être une initiation.

À la fin de cette analyse, nous pouvons conclure que chaque traducteur a interprété et représenté différemment le poème original, en rendant dominantes des isotopies qui n'avaient pas la même teneur ou le même statut dans le poème de départ, ou bien en renonçant à d'autres. Cette «variation» montre qu'il y a eu, dans la traduction, plus qu'une compréhension linguistique du texte source, plus précisément que la traduction a été une interprétation symbolique du poème rimbaldien, interprétation influencée par l'univers socioculturel et historique du traducteur qui projette dans la culture d'accueil une certaine représentation du poète symboliste et de son œuvre.

\section{CONCLUSION}

Texte poly-isotopique, «Les Chercheuses de poux» met en lumière la variation de l'effet du texte sur le lecteur/le traducteur et révèle la participation du lecteur/ traducteur à la production du sens du texte. L'analyse des isotopies de l'original et de ses traductions n'a pas visé de critiquer certaines traductions et d'en approuver d'autres, mais de montrer qu'il est essentiel pour un traducteur d'essayer de comprendre les manifestations isotopiques dans le texte qu'il traduit pour avoir ainsi plusieurs techniques de lecture.

Cette analyse a été aussi une occasion de mieux replacer le texte de Rimbaud dans la pluralité des discours de son époque, de comprendre et de recréer le langagesystème de l'auteur, c'est-à-dire l'ensemble de thèmes et de symboles qui assurent l'unité et l'originalité de son œuvre et qui conditionnent le processus de traduction. La notion d'isotopie nous a permis de faire, par l'entremise des quelques traductions, une suite de lectures possibles qui montrent qu'un texte, une traduction ne reste jamais au niveau du «cosmos», des "propositions factuelles», mais récupère l'impensé de l'auteur et de son époque, le silence qui se cache entre les mots. 


\section{NOTES}

1. M. Foucault, The Archaelogy of Knowledge \& The Discourse on Language, traduit du français par A.M. Sheridan Smith, New York / Hagerstown / San Francisco / Londres, Harper Colophon Books, Harper \& Row, 1976, p. 25.

2. E. Pound, Translations, Londres, Faber and Faber, 1953.

3 E.R. Peschel, Four French Symbolist Poets: Baudelaire, Rimbaud, Verlaine, Mallarmé, Athènes, Ohio University Press, 1981, p. 199.

4. J. Reed, Delirium, Grande Bretagne, Éd. Peter Owen, 1991, p. 130.

5. <http://www.geocities.com/Paris/Parc/3414/seekers.html >.

6. J. Theobald, The Lost Wine. Seven Centuries of French into English Lyrical Poetry, La Jolla, California, Green Tiger Press, 1980, p. 333.

7. R. Lowell, Imitations, NewYork, Farrar, Straus and Cudahy, 1961, p. 91.

8. Le «cosmos» se réfère à tout ce qui relève du monde, de la réalité représentée dans le texte. C'est le premier niveau de l'énonciation qui correspond, d'après P. Ouellet ( «La vision des choses: la focalisation dans le discours scientifique», Protée, Langage et Savoir, vol. 19, n 1, printemps 1985, p. 37), à «l'univers des objets focalisés» et qui regroupe «les 'états de choses' auxquels renvoient les propositions qu'on a l'habitude d'appeler factuelles, c'est-à-dire celles qui se présentent comme 'images propositionnelles' des faits de base ou des 'objets premiers du discours', sur lesquels s'appuie l'argumentation ou la narration prise en charge par l'énonciateur".

9. Un fragment du poème «L'Impossible» de Rimbaud nous fournit une autre explication allégorique du titre «Les Chercheuses de poux». Dans «L'Impossible» - qui reprend les isotopies de l'amour, de la femme, de l'impureté et de la pureté —, l'enfant rejette la société pourrie, où seuls les «parasites» ont le bonheur; l'enfant trouve, comme Mallarmé, que le réel est «vil»: «J'ai eu raison de mépriser ces bonshommes qui ne perdraient pas l'occasion d'une caresse, parasites de la propreté et de la santé de nos femmes, aujourd'hui qu'elles sont si peu d'accord avec nous» (nous soulignons). $\mathrm{La}$ «Chercheuse de poux» serait le symbole de la femme qui s'adonne aux multiples plaisirs de la vie quotidienne.

10. «Ancien instrument composé de récipients de verre plus ou moins remplis d'eau, que l'on faisait résonner par frottement ou en frappant avec des maillets» (dans J. Rey-Debove et A. Rey [dirs.], Le Nouveau Petit Robert. Dictionnaire alphabétique et analogique de la langue française, Paris, Dictionnaires Le Robert, 1996).

11. Dans un autre poème de Rimbaud, à savoir «Les Sœurs de charité», nous pouvons lire: «Alors, et toujours beau, sans dégoût du cercueil, / Qu'il croie aux vastes fins, Rêves ou Promenades / Immenses, à travers les nuits de Vérité / Et t'appelle en son âme et ses membres malades / Ô Mort mystérieuse, ô sœur de charité», ce qui nous donne une autre interprétation de la Femme: elle est vue ici comme désir de mourir, ou comme la Mort même.

\section{RÉFÉRENCES}

Arrivé, M. (1976) : Lire Jarry, Bruxelles, Éditions Complexe.

Barthes, R. (1970): S/Z, Paris, Éd. du Seuil.

Barthes, R. (1973): Le Plaisir du texte, collection «Tel Quel», Paris, Éd. du Seuil.

BAyo, G. (1987): Arthur Rimbaud, Troyes, Éd. Librairie bleue.

Eliot, T.S. (dir.) (1954): Literary Essays of Ezra Pound, Grande Bretagne, New Directions.

Eliot, T.S. (1990): On Poetry and Poets, Londres et Boston, Faber and Faber.

Foucault, M. (1976): The Archaelogy of Knowledge \& The Discourse on Language, traduit du français par A.M. Sheridan Smith, New York / Hagerstown / San Francisco / Londres, Harper Colophon Books, Harper \& Row.

Greimas, A.J. (1966) : «Réflexions sur les modèles actantiels», dans Sémantique structurale, Paris, Larousse, p. 172-201.

Iser, Wolgang (1985): L'acte de lecture. Théorie de l'effet esthétique, trad. de l'allemand par Evelyne Sznycer, Liège/Bruxelles, Pierre Mardaga.

Lowell, R. (1961): Imitations, NewYork, Farrar, Straus and Cudahy.

Mallarmé, S. (1974): CEuvres complètes, Paris, Gallimard. 
Mailloux, S. (1995): «Interpretation», dans F. Lentricchia et T. McLaughlin [dirs.], Critical Terms for Literary Study, Chicago, The University of Chicago Press, 121-134.

Meschonnic, H. (1970): «D’une linguistique de la traduction à la poétique de la traduction », dans Pour la poétique II, Épistémologie de l'écriture. Poétique de la traduction, Paris, Gallimard, 327-366.

Ouellet, P. (1985): «La vision des choses: la focalisation dans le discours scientifique», dans Protée, Langage et Savoir, vol. 19, $\mathrm{n}^{\circ}$ 1, printemps 1985, 33-45.

Peschel, E. R. (1981): Four French Symbolist Poets: Baudelaire, Rimbaud, Verlaine, Mallarmé, Athènes, Ohio University Press.

Plouvier, P. (1996): Sous la lumière de Nietzsche: Rimbaud ou le corps merveilleux, Saint-Maximin, Éd. Théétète.

Pound, E. (1953): Translations, Londres, Faber and Faber.

Rastier, F. (1972) : «Systématique des isotopies», dans A.J. Greimas (dir.), Essai de sémantique poétique, Paris, Larousse, p. 80-106.

Reed, J. (1991): Delirium, Grande Bretagne, Éd. Peter Owen.

Rey-Debove, J. et A. Rey [dirs.] (1996): Le Nouveau Petit Robert. Dictionnaire alphabétique et analogique de la langue française, Paris, Dictionnaires Le Robert.

Rimbaud, A. (1992): CEuvres complètes. Correspondance, Paris, Éd. Robert Lafont.

Theobald, J. (dir.) (1980): The Lost Wine. Seven Centuries of French into English Lyrical Poetry, La Jolla, California, Green Tiger Press.

Whitaker, M.-J. (1972): La structure du monde imaginaire de Rimbaud, Paris, Éd. A.-G. Nizet.

Webster's New World Dictionary of American English, Cleveland et New York, Éd. Third College, 1991. 